\title{
Surgical Placement of Permanent Pacemaker Following Temporary Trasvenous Pacemaker in a Child with Congenital Heart Block and Moderate Size PDA: A Case Report
}

\author{
KAZI ZAHIDUL HOQUE ${ }^{1}$, MASUMUL GANI CHOWDHURY ${ }^{2}$, ABU SAYEED MUNSI ${ }^{3}$, REZOANA RIMA ${ }^{3}$, \\ MANZOOR HUSSAIN 4
}

\section{Introduction}

Congenital complete atrioventricular block (CCAVB), with incidence of $1 / 15000-20000$ live born, is characterized by no transmission of any atrial electrical impulses to ventricles. It is almost always due $(90 \%$ of cases) to the transfer of auto-antibodies (SSA/Ro and SSB/La) from SLE - or Sjogren's syndrome- affected mothers, even during their preclinical phase of the disease. ${ }^{1-3}$ These auto-antibodies are responsible of ventricular endocardium damage and subsequent endomyocardialfibroelastosis. This can cause intrauterine miscarriage, with underestimation of the real incidence of the disease or, fetal third-degree block, prolonged QT and Wolff ParkinsonWhite syndrome. ${ }^{2}$ Third-degree block is frequently diagnosed during pregnancy, around 16-18 weeks of gestation. Timing of delivery as well as type and time of pacemaker implantation after birth are still controversial issues. Pacemaker implantation is indeed the only treatment for third-degree block and it is immediately required in presence of prenatal hydrops, low ventricular rate $(<45 / \mathrm{bpm})$ with no response to inotropes, and/or ventricular dysfunction. ${ }^{3}$ In very low birth weight infants, because of the size of generators, a staged pacing strategy has been used, with temporary epicardial pacing wires, followed by definitive implantation when neonatal body weight reaches $2000 \mathrm{~g}$. Some cases have also been treated with permanent pacemaker implantation. ${ }^{3-5}$

1. Assistant Professor, Pediatric Cardiac Surgery, Dhaka Shishu (Children) Hospital

2. Assistant Professor, Pediatric Cardiac Surgery, Dhaka Shishu (Children) Hospital

3. Assistant Professor, Pediatric Cardiology \& Interventional cardiology, Dhaka Shishu (Children) Hospital

4. Assistant Professor, Pediatric Cardiology \& Interventional cardiology, Dhaka Shishu (Children) Hospital

5. Director and Professor and Departmental Head, Pediatric Medicine \& Pediatric Cardiology, Dhaka Shishu (Children) Hospital

Correspondence: Dr. KaziZahidulHoque, Assistant Professor, Pediatric Cardiac Surgery, Dhaka Shishu (Children) Hospital, Sher-e-Bangla Nagar, Dhaka, E-mail: kzhoque72@yahoo.com

\section{Case Report}

A 9-months-old female child, born with the history of parents consanguineous marriageby caesarian section in a hospital with a birth wei-ght of $3.3 \mathrm{~kg}$, presented with bradycardia (heart rate of $30-35$ beats/ min) and respiratory distress and with multiple syncopal attacks since her 8 months of age, for which she was admitted to Paediatric Cardiology unit of Dhaka Shishu (Children) Hospital. His mother, Gravida 2 and Para2, had no past medical history significant for Systemic Lu-pus Erythematosusor Sjogren syndrome. Her previous baby was in good health and no major congenital anomaly present. She has reported that the baby was prenatally diagnosed bradycardic by ultrasonogram and they were informed about the condition of the fetus. The parents also reported a history of repeated syncopal attacks and respiratory tract infections since the age of one month. Prior to that the child under treatment with diuretics and captopril and antibiotics.Clinical examination showed a continuous machinery murmur $4 / 6$ at the left $2^{\text {nd }}$ intercostal space. Femoral pulses weresymmetrically palpable. The infant was adequately nourished. Her psychomotor development was normal according to her chronological age. The first ECG showed (Fig.-2)3 ${ }^{\text {rd }}$ degree heart block with complete arioventricular dissociation. The child was stabilized in the cardiology unit and kept on two liters of oxygen through nasal cannula for respiratory distress. She was tolerating feeds. Her heart rate ranged between $30-35$ beats/ min despite being given two doses of atropine $(0.1 \mathrm{mg} /$ dose $)$. Chest $X$-ray AP view showed increased diameter of the cardiac shadow(Fig.-1). Her electrocardiogram (ECG) showed features of complete heart block (Fig.-2), with the features of complete atrioventricular dissociation and heart rate was $34 / \mathrm{min}$. Based on her condition, it was decided to do further evaluation and management (including the possi-bility of pacemaker insertion) to support the patient in the best possible way. 


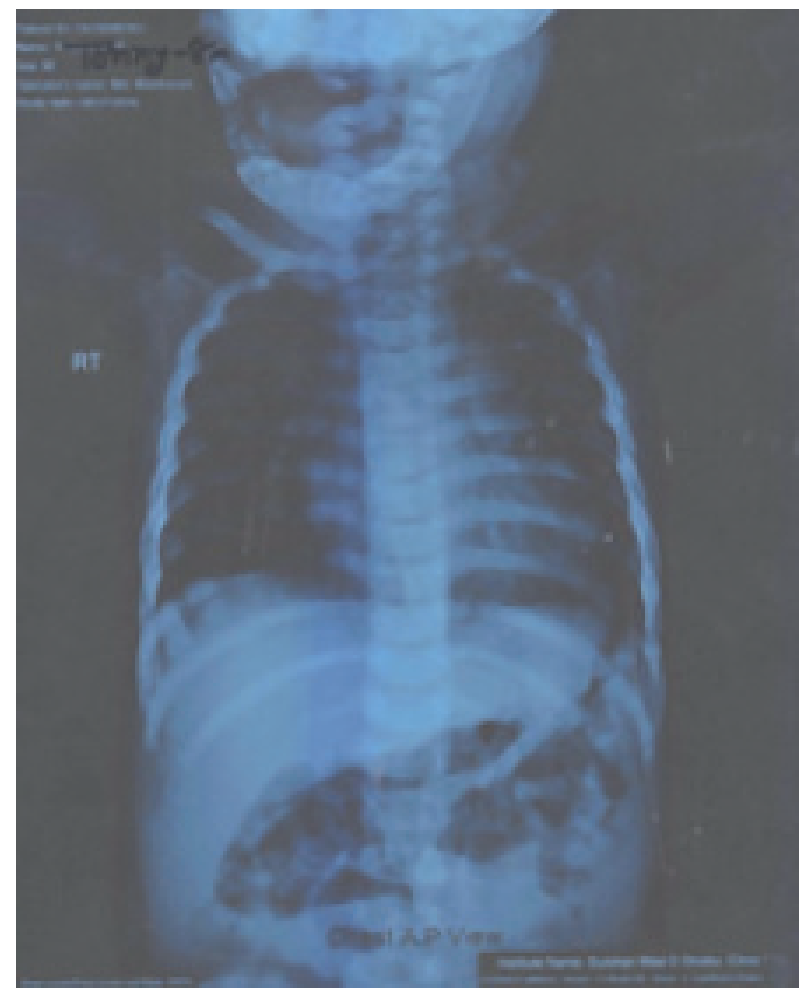

Fig.-1: Preoperative X Ray Shows increased Cardiac Shadow and Plethoric Lung field

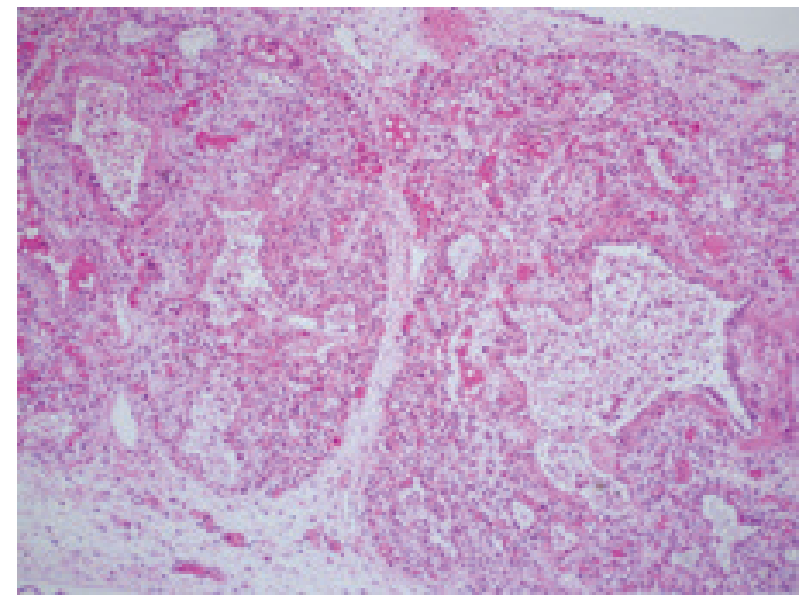

Fig.-2: ECG before placing any pacemaker shows $3^{\text {rd }}$ degree heart block

Echocardiography was performed and it revealed a PFO with moderate size PDA shunting left to right. Rate showed extreme bradycardiac, no other abnormality detected.Considering the emergency situation intervention cardiology unit decided to put a temporary transvenous pacemaker. They ultimately implanted it on the $4^{\text {th }}$ day of admission through the right femoral vein route and placed it into the right ventricular apex under general anesthesia, and the other end of the lead was connected to an external pacemaker generator and set the rate at $90 \mathrm{~b} / \mathrm{min}$ with output $5 \mathrm{~mA}$ and capture was lost in $1 \mathrm{~mA}$. The patient had developed repeated ventricular tachycardia (VT) during the procedure but no neurological abnormality detected after the procedure. We the cardiac surgery team decided to do a left lateral thoracotomy. Through that incision we ligated the PDA and implanted a permanent VVI pace maker at the apex of the heart. The decision of lateral thoracotomy was made on the basis of convenience for the PDA ligation otherwise it could be done through median sternotomy.At $7^{\text {th }}$ admission day upon the availability of permanent pacemaker, baby was taken to operation theater, a left lateral thoracotomy was made at

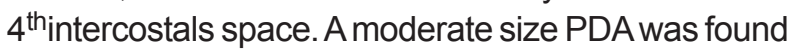
after dissection of the fascia over the aorta. Multiple ligatureswere made to close the PDA (Fig.-5 \&6). Pericardium was incised with a caution forpericardiacophrenic nerve and vessels. A permanent pacemaker (St. Jude VVIR PM with autocapture at rate 90 beats $/ \mathrm{min}$ ) implantation was done and set the rate at $90 \mathrm{~b} /$ minutes and the generator was placed in the abdomen(Fig.-7 \& 8) after making a subcutaneous pocket. Chest $X$ ray (Fig. 4 ) shows the placement of lead and generator. The temporary pacemaker was disconnected as soon as commencement of the permanent pacemaker. A 12 lead ECG was done (Fig.-9) in the postoperative recovery unit. The ECG shows heart rate $90 \mathrm{~b} / \mathrm{min}$ with frequent pacemaker capture beats. Upon further follow up, the patient was stable and his general health was good. He was thriving well with normal developmentin subsequentfollow-up. The echocardiographic examination showed good left ventricular contractility with normal internal dimensions. There was no residual flow through the closed PDA.

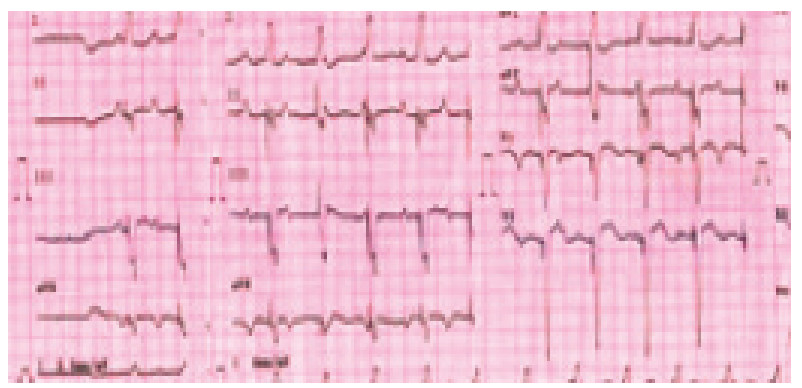

Fig.-3: ECG after placing temporary pace maker. Heart rate shows 90b.min and Pacemaker capture is visible 


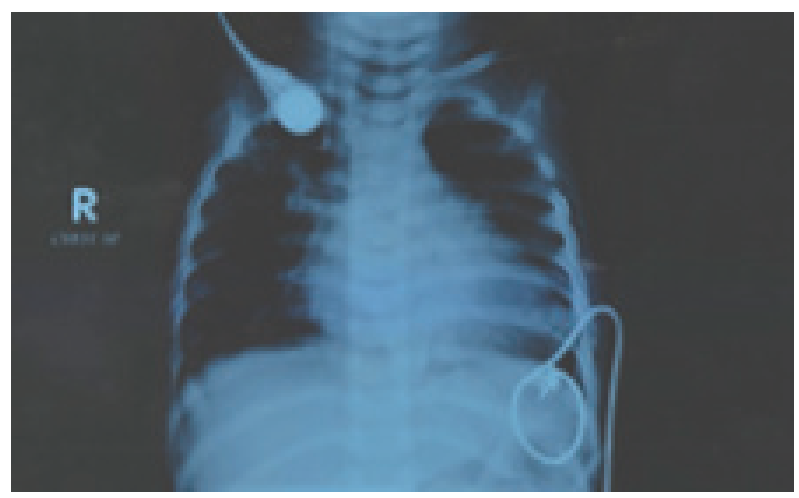

Fig.-4: Chest $X$ ray after surgical implantation of permanent pacemaker

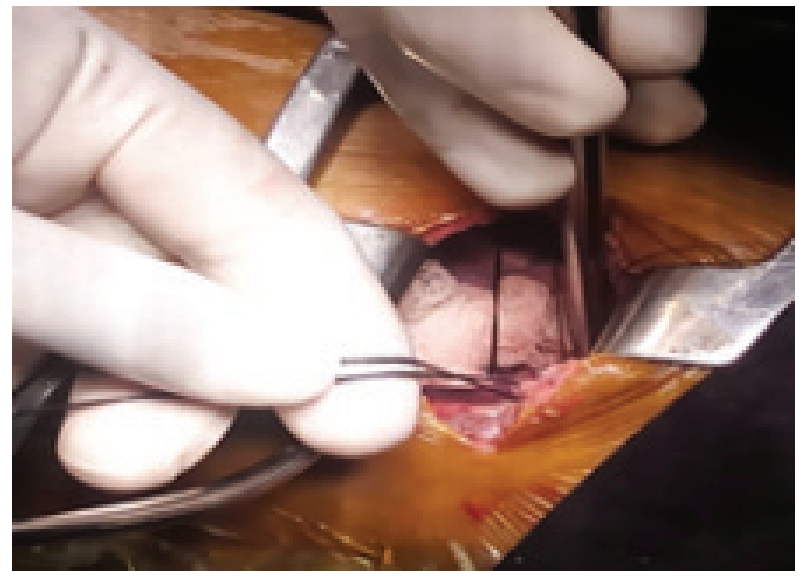

Fig.-6: Multiple ligation of PDA

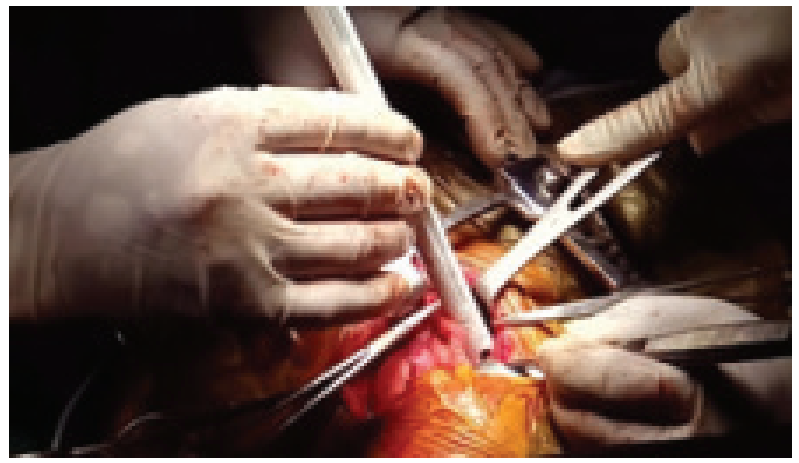

Fig.-8. Pacemaker Implantation through lataral thoracotomy

\section{Discussion}

It is well known that in both infants and older children permanent pacemaker leads may be implantedeither epicardially or transvenously. In small infants, when permanent pacemaker implantation is necessary epicardial leads are used. The reason for preferring

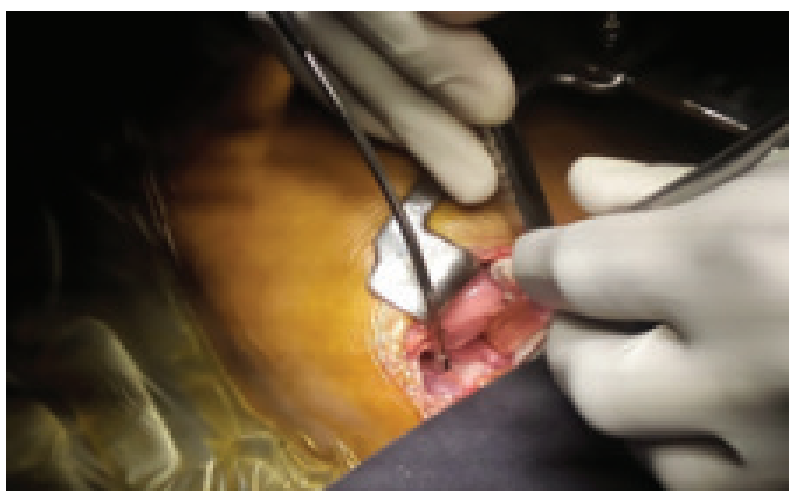

Fig.-5: Lateral thoracotomy for PDA ligation \& pacemaker implantation

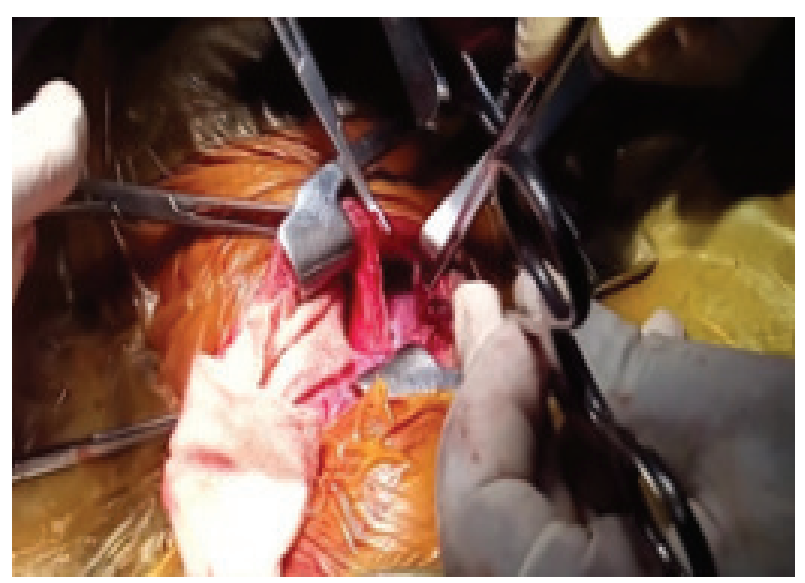

Fig.-7. Incision through pericardium

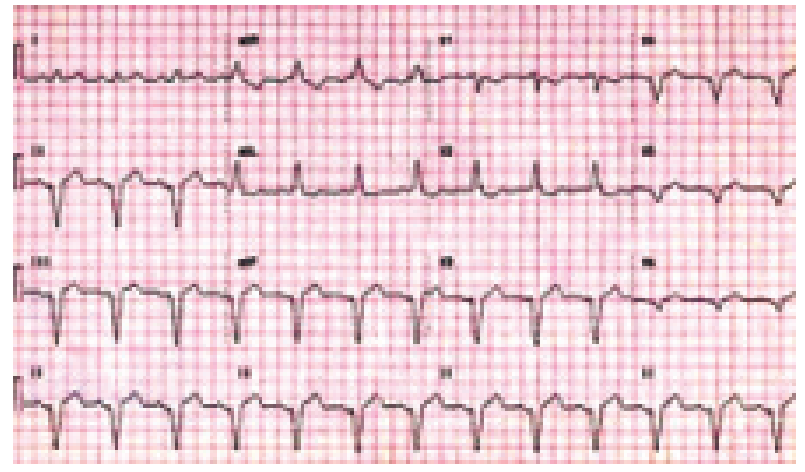

Fig.-9: ECG after permanent pacemaker implantation. Hear rate is $90 \mathrm{~b} / \mathrm{min}$ and pacemaker capture is visible

the epicardial route is the patient's small body size. Transvenous lead implantation is hampered by anatomical peculiarities, which are often seen and include anomalous connection of the venae cavaeas well as other complex endocardial anatomical lesions. ${ }^{6}$ Apart from the procedural difficulties, such anomalies 
also entail a risk of systemic embolism due to endocardial defects. ${ }^{7}$ In addition, thrombosis in the superior vena cava is a common complication of endocardial lead placement ${ }^{8}$

On the other hand, epicardial lead placement is more invasive. It involves subxiphoid section and possibly a partial sternotomy or thoracotomy. It is often complicated by post-pericardiotomy syndrome. ${ }^{9}$ The usual epicardial leads are associated with a high incidence of rapidly increasing sensing and pacing thresholds after lead placement, necessitating the early replacement of lead and generator. Endocardial leads are favoured inolder infants with a body weight $>8 \mathrm{~kg},{ }^{10}$ or preferably $15-20 \mathrm{~kg},{ }^{11}$. Moreover, insmall infants the small dimensions of the atrium ${ }^{11}$ areinsufficient for successful placement of the preformedatrial lead. The elevated pacing thresholds and the high incidenceof exit block associated with conventional epicardialleads are caused by a combination of epicardial fibrosiswith scar formation, and/or pericardial adhesionsfollowing the surgical procedure. Cases havebeen reported of exit block due to lead fracture caused by the infant's muscular activity. ${ }^{12}$ The five-year survival of the conventional epicardial lead is $40-70 \% .{ }^{13,14}$ In neonates and infants with a permanent pacemakerthe occurrence of episodes of loss of consciousnessmay be due to pacemaker malfunction. ${ }^{15}$ Follow-upchecks should be performed every 6 months inthose without symptoms; parameters of pacemakerfunction should be measured, mainly the resistanceand threshold of the atrial and ventricular leads. Ifsymptoms occur as a result of pacemaker malfunction,24-hour Holter monitoring is useful for their detection. ${ }^{16}$ Pacemaker malfunction due to the developmentof fibrosis around one or both of the epicardial leadscan be treated by the substitution of endocardial leads. At the same time, the generator can be left in its abdominalsite and the electrodes can be connected viaa subcutaneous channel.

\section{Conclusion}

In conclusion, the choice of lead type during pacemaker implantation should aim at achieving optimum cardiac function and maximum battery and lead life, while taking account of the risks of lead placement as well as the future surgical treatment of the patient.

\section{References}

1. Kratz JM, Gillette PC, Crawford FA. Atrioventricular pacing in congenital heart disease. Ann Thorac Surg. 1992;54: 485-89.

2. Weindling SN, Saul JP, Gamble WJ, Mayer JE, Wessel D, Walsh EP. Duration of complete atrioventricular block aftercongenital heart disease surgery.Am J Cardiol.1998; 82: 525-27.

3. Kertesz N, McQuinn T, Collins E. Surgical atrioventricular block in 888 heart operations. New implications for earlyimplantation of a permanent pacemaker.Pacing Clin Electrophysiol.1996; 19: 613.

4. Udink ten Cate F, Breur J, Boramanand N. Endocardial and epicardial steroid lead pacing in the neonatal and pediatric age group. Heart.2002; 88: 392-96.

5. Noiseaux N, Khairy P, Fournier A, Vobecky SJ. Thirty years of experience with epicardial pacing in children. Cardiol Young. 2004; 14: 512-19.

6. Aellig NC, Balmer C, Dodge-Khatami A, Rahn M, Prêtre R, Bauersfeld U. Long-term follow-up after pacemaker implantation in neonates and infants. Ann Thorac Surg. 2007: 83; 1420-23.

7. Beaufort-Krol GC, Mulder H, Nagelkerke D, Waterbolk TW, Bink-Boelkens MT. Comparison of longevity, pacing and sensing characteristics of steroid eluting epicardial versus conventional endocardial pacing leads in children. $J$ ThoracCardiovasc Surg. 1999; 117: 523-28.

8. Gillette PC, Shannon C, Blair $\mathrm{H}$, et al. Transvenous pacing in pediatric patients. Am Heart J. 1983; 10: 843-47.

9. Hamilton R, Gow R, Bahoric B. Steroid-eluting epicardial leads in pediatrics: improved epicardial thresholds in the first year. Pacing ClinElectrophysiol.1991; 14: 2066-72.

10. Johns JA, Fish FA, Burger JD, Hammon JW Jr. Steroid-eluting epicardial pacing leads in pediatric patients: encouraging early results. J Am CollCardial. 1992; 20: 395-401.

11. Cohen MI, Bush DM, Vetter VL. Permanent epicardial pacing in pediatric patients: seventeen years of experience and 1,200 outpatient visits.Circulation.2001; 103: 2585-90. 
12. Silveti MS, Drago E. Upgrade of single chamber pacemakers with transvenous leads to dual chamber pacemaker in pediatric and young adult patients. Pacing ClinElectrophysiol.2004; 27: 1094-98.

13. Silvetti MS, Drago F, Grutter G. Twenty years of paediatric cardiac pacing: 515 pacemakers and 480 leads implanted in 292 patients. Europace.2006; 8: 530-36.
14. Bakhtiary F, Dzemali O, Bastanier CK. Mediumterm follow-up and modes of failure following epicardial pacemaker implantation in young children. Europace.2007; 9: 94-97.

15. Sachweh JS, Vazquez-Jimenec JF, Schöndube FA. Twenty years experience with pediatric pacing: epicardial and transvenous stimulation. Eur J Cardiothorac. Surg. 2000; 17: 455-461.

16. Kratz JM, Gillette P C, Crawford FA. Atrioventricular pacing in congenital heart disease.Ann Thorac Surg. 1992; 54: 485-89. 